\title{
Evaluación posalta del crecimiento en prematuros. Implicaciones de adoptar las curvas OMS
}

\author{
Post discharge growth assessment in preterm infants. \\ Implications of adopting the WHO standards
}

\author{
Dr. Carlos A. Fustiñana ${ }^{a}$, Dra. Diana Rodríguez y Dr. Gonzalo Mariani ${ }^{a}$
}

\section{RESUMEN}

Introducción. Los estándares para evaluar el crecimiento en los prematuros se han utilizado como referencia desde el año 1986. La introducción de las curvas OMS en la Argentina podría incrementar la detección de los niños con crecimiento subóptimo.

Objetivo. Comparar la proporción de retraso del crecimiento en peso, longitud corporal y perímetro cefálico en prematuros con peso al nacer menor de $1500 \mathrm{~g}$ (RNMBPN) evaluados por las curvas OMS y las curvas SAP.

Pacientes y métodos. Estudio de cohorte en RNMBPN. Se incluyeron las mediciones de peso, longitud corporal y perímetro cefálico, realizadas a las 40, 53, 66, 79 y 92 semanas posmenstruales ( \pm 1 semana). Se registró como variable independiente el sexo para ambas curvas (OMS y SAP). Se analizaron las medias del puntaje $Z$ para ambas curvas mediante la prueba deStudent y la diferencia de proporciones mediante la prueba de la $\chi^{2}$ (OR; IC $95 \%$ ).

Resultados. Ingresaron 204 niños. Las variables antropométricas al nacer divididas por sexo no mostraron diferencias. El peso y la longitud mostraron unaapreciación mayor del crecimiento por las curvas SAP vs. OMS, más en los varones que en las mujeres, y se atenuaron al año de edad. La estatura evaluada porOMS presentó retrasoen ambos sexos. La circunferencia craneal no arrojó diferencias. Seencontróuna mayor proporción de pacientes con peso por debajo de 2 desviaciones estándar a los 3 meses de edad según OMS $(p<$ 0,01 ; OR 0,36; IC $95 \%$ 0,15 a 0,78), no así para la longitud y el perímetro cefálico.

Conclusiones. Este estudio permite sugerir que el cambio de estándar no implicaría una modificación significativa a nuestras prácticas de seguimiento durante el primer año de edad.

a. Servicio de

Neonatología, Hospital Italiano de Buenos Aires.

Correspondencia:

Dr. Carlos A. Fustiñana carlos.fustinana@ hospitalitaliano.org.ar

Conflicto de intereses: Ninguno que declarar.

Recibido: 13-6-2013 Aceptado: 4-9-2013
Palabras clave: prematuridad, crecimiento posnatal, estándar OMS, estándar SAP.

http:/ /dx.doi.org/10.5546/aap.2014.141

\section{INTRODUCCIÓN}

Los estándares para evaluar el crecimiento hasta la semana 92 de edad posmenstrual se publicaron en el año 1986; ${ }^{1}$ desde entonces, dichas curvas se han utilizado como referencia para evaluar el crecimiento posnatal de recién nacidos de pretérmino $y$, en especial, de aquellos con peso al nacer menor de 1500 g. ${ }^{2}$ La practicidad de las curvas permitió el amplio uso por los grupos de seguimiento de prematuros de muchas instituciones nacionales, fueron recomendadas por el CEFEN de la Sociedad Argentina de Pediatría ${ }^{3}$ y mostraron, en estudios posteriores, que tanto la restricción del crecimiento intrauterino como la morbilidad neonatal ejercían efectos perjudiciales sobre el crecimiento posnatal ${ }^{4}$ durante el primer año de vida.

Desde su publicación hasta la fecha se han producido numerosos cambios en las estrategias del cuidado neonatal, como el uso de surfactante pulmonar exógeno, estrategias ventilatorias (ventilación no invasiva y de alta frecuencia), implementación de paquetes de medidas (bundles) para mejorar la nutrición y disminuir la infección intrahospitalaria, los cuales produjeron mejoras de la eficacia, que repercutieron sobre la morbimortalidad y modificaron los límites de la viabilidad. ${ }^{5}$ Estas estrategias también han mejorado el resultado del crecimiento de estos pacientes, aunque aún sin alcanzar las metas esperadas.

En abril de 2006, la OMS dio a conocer nuevos patrones del crecimiento infantil para evaluar el crecimiento de esta población hasta los 5 años de edad. ${ }^{6}$ Esto permitió el reemplazo del patrón del National Center for Health Statistics (NCHS), que no era aceptado en muchos países, entre ellos, la Argentina. 
El criterio utilizado para la construcción del nuevo estándar permitió mostrar que un niño sano, independientemente de su lugar de origen, si se cuida su ambiente (libre de humo), se realizan controles pediátricos periódicos, se cumple con la vacunación y se siguen similares prácticas nutricionales (alimentación específica por los primeros seis meses de vida), expresará similares patrones de crecimiento. La aparición de este estándar propuesto por la OMS es prescriptivo (así deberían crecer los niños), en vez de los descriptivos que habitualmente usamos para controlar el crecimiento de los pacientes.

Las curvas propuestas por OMS fueron validadas en nuestro país en una población de 0 a 5 años en la ciudad de Rosario. ${ }^{7}$ Esto llevó a su implementación en la Argentina. Dicha circunstancia nos ha llevado a preguntarnos si su aplicación sobre una población de riesgo, como la de los prematuros de muy bajo peso al nacer, implicaría incrementar la detección de niños con crecimiento subóptimo durante el primer año de edad corregida.

El objetivo de este estudio fue comparar si la proporción de retardo del crecimiento posnatal en peso, longitud corporal y perímetro cefálico, en prematuros con peso al nacer menor de $1500 \mathrm{~g}$, es distinta entre las curvas propuestas por la OMS, en lugar de las utilizadas hasta la fecha (curvas $\mathrm{SAP}$ ) en el seguimiento durante el primer año de vida.

\section{PACIENTES Y MÉTODOS}

Se trató de un estudio de cohorte retrospectivo, con datos tomados de una base de datos activa de seguimiento de prematuros con peso al nacer menor de $1500 \mathrm{~g}$, completada en forma prospectiva. Se incluyeron las mediciones de peso, longitud corporal y perímetro cefálico, realizadas a las 40,53, 66, 79 y 92 semanas posmenstruales ( \pm 1 semana). Las mediciones de peso se realizaron con balanza de palanca y se contabilizó hasta los 10 g completos. Para las mediciones de longitud y perímetro cefálico se registró hasta el último milímetro completo y las realizó uno de los autores (DR) de acuerdo con las recomendaciones del Comité de Crecimiento y Desarrollo de la SAP. Se registró como variable independiente el sexo, dado que ambas curvas (OMS y SAP) dividen a la población en niñas y niños.

Se incluyeron todos los pacientes que presentaban al menos tres mediciones en las fechas indicadas. También se incluyeron los niños con restricción del crecimiento intrauterino o morbilidad grave por considerar que no modificarían el resultado de la evaluación. Se excluyeron los pacientes con malformaciones mayores. Se consignó el peso al nacer, la edad gestacional, la edad corregida al alta, el peso, la longitud corporal y el perímetro cefálico al alta.

Se calcularon las desviaciones medias mediante el cálculo del puntaje de desvío de $\mathrm{Z}$ a las $40,53,66,79$ y 92 semanas posmenstruales $( \pm 1$ semana) para cada una de las curvas estudiadas (SAP y OMS). Las desviaciones se evaluaron mediante la prueba de Student. Las diferencias de proporciones de niños/as por debajo de $2 \mathrm{DE}$ según cada curva (SAP y OMS) se compararon mediante la prueba de la $\chi^{2}$ y se calcularon los odds ratios y los intervalos de confianza del $95 \%$. Se calculó un tamaño muestral de 135 pacientes para hallar al menos $10 \%$ de los pacientes por debajo de $2 \mathrm{DE}$ del peso de diferencia entre OMS o SAP y un OR $\leq 0,2$ para una potencia de $80 \mathrm{y}$ un intervalo de confianza del $95 \%$.

\section{RESULTADOS}

Durante el período 2007-2010 ingresaron en el programa de seguimiento 271 niños con peso al nacer inferior a $1500 \mathrm{~g}$. No cumplieron con tres mediciones en las fechas requeridas 58 pacientes, 9 presentaron malformaciones congénitas mayores ( 5 cardiopatías aisladas y 4 síndromes cromosómicos o genéticos). Cumplieron con los criterios de inclusión 204 niños, que fueron los analizados en el estudio. En la Tabla 1 se muestran las características del grupo divididas por sexo. No se apreciaron diferencias en el peso al nacer ni en la edad gestacional. Las características antropométricas y la edad corregida en el momento del alta también fueron similares.

TABLA 1. Media y desviación estándar al nacer y al alta del grupo de estudio y proporción de bajo peso al nacer, según el sexo

\begin{tabular}{lcc}
\hline & \multicolumn{2}{c}{ Media \pm DE } \\
\hline & Niños (101) & Niñas (103) \\
\hline Peso al nacer (g) & $1160 \pm 237$ & $1110 \pm 247$ \\
Edad gestacional (sem.) & $29,4 \pm 2,8$ & $29,2 \pm 2,7$ \\
Peso al alta (g) & $2466,9 \pm 622$ & $2467,2 \pm 607$ \\
Longitud al alta (cm) & $43,9 \pm 2,9$ & $43,8 \pm 4,3$ \\
PC al alta (cm) & $33,4 \pm 2,5$ & $33,1 \pm 2$ \\
Edad corregida al alta (sem.) & $38,3 \pm 4,6$ & $38,1 \pm 4,5$ \\
Bajo peso para EG $n$ (\%) & $41(41)$ & $42(40)$ \\
\hline
\end{tabular}

PC: perímetro cefálico.

EG: edad gestacional. 
En la Tabla 2, se muestran las medias y las desviaciones estándar de las tres variables antropométricas divididas por sexo y expresando su desviación frente a las medias de las tablas OMS y SAP. Tanto el peso como la longitud mostraron diferencias entre la apreciación mayor del crecimiento por las curvas SAP respecto de las curvas OMS. Estas fueron máximas a las 53 y a las 66 semanas para el peso, más marcadas en los varones (Figura 1) que en las mujeres y se atenuaron al llegar al año de edad posmenstrual. La estatura evaluada por OMS también presentó un retraso en ambos sexos, aunque más acentuado en las niñas, que se extendió desde la semana 53 hasta la 79 inclusive (Figura 2). El seguimiento de la circunferencia craneal no arrojó diferencias evaluado por las dos curvas.
Al analizar las proporciones de pacientes con peso por debajo de $2 \mathrm{DE}$ (Tabla 3 ) según ambas referencias, a los 3 meses de edad corregida (53 semanas), las curvas SAP mostraron una menor proporción de pacientes por debajo de $2 \mathrm{DE}$ ( $\mathrm{p}$ $<0,01$; OR 0,36; IC 95\% 0,15 a 0,78). Al analizar la longitud corporal y el perímetro cefálico las diferencias no alcanzaron significación estadística.

\section{DISCUSIÓN}

El crecimiento posnatal de los RNMBPN es modificado por la presencia de restricción del crecimiento intrauterino, así como por la morbilidad posnatal. La lesión prenatal implica agresión en períodos críticos del desarrollo, con implicaciones profundas en la programación epigenética e impacto, incluso en la vida adulta.

TABla 2. Media y desviación estándar de las tres variables antropométricas divididas por sexo con los puntajes Z comparadas con las medias de las tablas OMS y SAP

\begin{tabular}{|c|c|c|c|c|c|c|c|}
\hline & $\begin{array}{c}\text { Edad } \\
\text { corregida }\end{array}$ & Sexo & $n$ & Media \pm DE & $\mathrm{Z}$ (SAP) & Z (OMS) & $\Delta \mathrm{Z}$ \\
\hline \multirow{10}{*}{ Peso (g) } & \multirow{2}{*}{40 sem. } & Niñas & 103 & $2888 \pm 495$ & $-0,9$ & $-1,02$ & 0,3 \\
\hline & & Niños & 101 & $3026,1 \pm 532$ & $-0,8$ & $-1,1$ & $-0,3$ \\
\hline & \multirow[t]{2}{*}{$53 \mathrm{sem}}$. & Niñas & 65 & $5171,1 \pm 697$ & $-0,6$ & $-1,2$ & $-0,6$ \\
\hline & & Niños & 63 & $5550,6 \pm 922$ & $-0,4$ & $-1,2$ & $-0,8$ \\
\hline & \multirow[t]{2}{*}{66 sem. } & Niñas & 71 & $6634,6 \pm 942$ & $-0,11$ & $-0,86$ & $-0,75$ \\
\hline & & Niños & 73 & $7336 \pm 1113$ & $-0,44$ & $-0,8$ & $-0,36$ \\
\hline & \multirow[t]{2}{*}{79 sem. } & Niñas & 51 & $7680,9 \pm 1511$ & $-0,01$ & $-0,7$ & $-0,69$ \\
\hline & & Niños & 52 & $8356,1 \pm 1139$ & $-0,01$ & $-0,6$ & $-0,59$ \\
\hline & \multirow[t]{2}{*}{92 sem. } & Niñas & 94 & $8472,9 \pm 1160$ & $-0,33$ & $-0,4$ & $-0,07$ \\
\hline & & Niños & 96 & $9250,8 \pm 1456$ & $-0,76$ & $-0,6$ & 0,16 \\
\hline \multirow{10}{*}{$\begin{array}{l}\text { Longitud } \\
\text { corporal }(\mathrm{cm})\end{array}$} & \multirow[t]{2}{*}{40 sem. } & Niñas & 96 & $46,1 \pm 2,6$ & $-2,3$ & $-2,3$ & 0 \\
\hline & & Niños & 92 & $46,9 \pm 3,1$ & $-2,17$ & $-2,1$ & 0,07 \\
\hline & \multirow[t]{2}{*}{53 sem. } & Niñas & 60 & $56,6 \pm 2,8$ & $-1,25$ & $-2,0$ & $-0,75$ \\
\hline & & Niños & 58 & $58,0 \pm 3,4$ & $-2,1$ & $-1,9$ & 0,3 \\
\hline & \multirow[t]{2}{*}{66 sem. } & Niñas & 70 & $62,7 \pm 3,8$ & $-0,68$ & $-1,9$ & $-1,2$ \\
\hline & & Niños & 70 & $65,6 \pm 4,7$ & $-0,41$ & $-0,8$ & $-0,39$ \\
\hline & \multirow[t]{2}{*}{79 sem. } & Niñas & 50 & $68,1 \pm 3$ & $-0,42$ & $-0,8$ & $-0,38$ \\
\hline & & Niños & 52 & $69,6 \pm 3,9$ & $-0,48$ & $-1,1$ & $-0,62$ \\
\hline & \multirow[t]{2}{*}{92 sem. } & Niñas & 83 & $71,7 \pm 3,3$ & $-0,85$ & $-0,7$ & 0,05 \\
\hline & & Niños & 96 & $73,4 \pm 4,7$ & $-0,63$ & $-0,9$ & 0,37 \\
\hline \multirow{10}{*}{$\begin{array}{l}\text { Perímetro } \\
\text { cefálico }(\mathrm{cm})\end{array}$} & \multirow[t]{2}{*}{40 sem. } & Niñas & 91 & $34,6 \pm 1,7$ & 0 & 0,1 & 0,1 \\
\hline & & Niños & 86 & $34,9 \pm 1,4$ & $-0,36$ & 0,1 & 0,46 \\
\hline & \multirow[t]{2}{*}{53 sem. } & Niñas & 61 & $39,6 \pm 1,8$ & $-0,18$ & 0,12 & 0,3 \\
\hline & & Niños & 61 & $40,4 \pm 1,6$ & $-0,15$ & 0,02 & 0,17 \\
\hline & \multirow[t]{2}{*}{$66 \mathrm{sem}}$. & Niñas & 68 & $42,3 \pm 2,6$ & $-0,35$ & 0,01 & 0,36 \\
\hline & & Niños & 64 & $43,7 \pm 2,2$ & $-0,08$ & 0,1 & 0,18 \\
\hline & \multirow[t]{2}{*}{79 sem. } & Niñas & 50 & $44,4 \pm 1,6$ & $-0,20$ & 0,1 & 0,3 \\
\hline & & Niños & 51 & $45,1 \pm 1,8$ & $-0,40$ & 0,02 & 0,42 \\
\hline & \multirow[t]{2}{*}{92 sem. } & Niñas & 72 & $44,9 \pm 1,8$ & $-0,58$ & 0,1 & 0,68 \\
\hline & & Niños & 79 & $46 \pm 1,9$ & $-0,72$ & 0 & 0,72 \\
\hline
\end{tabular}


FIgURA 1. Curvas longitudinales donde se muestran los puntajes de desviación estándar del peso durante el primer año de vida. Se grafican los resultados divididos en mujeres y varones comparados con las curvas SAP y OMS

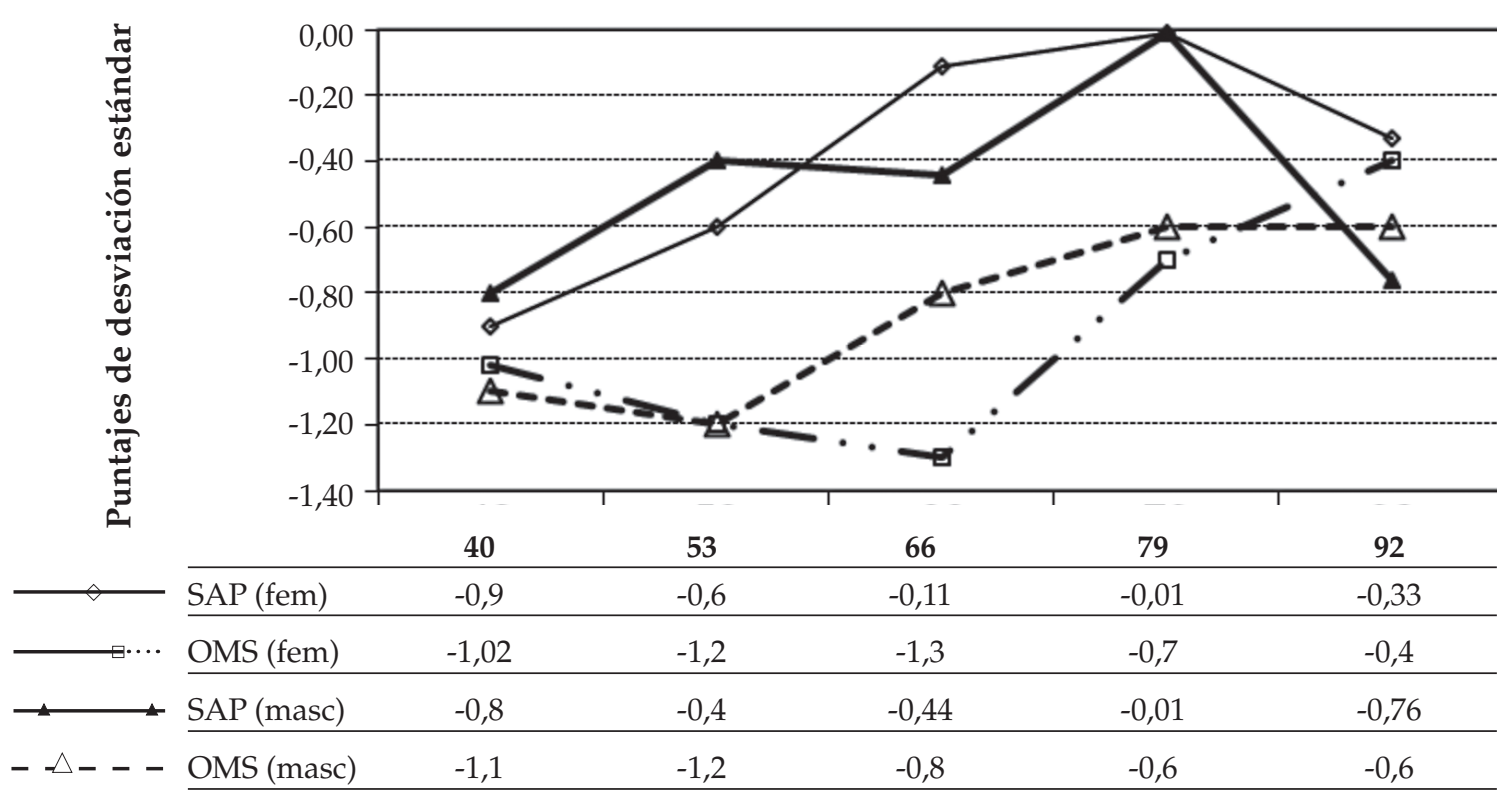

Edad posmenstrual

FIGURA 2. Curvas longitudinales donde se muestran los puntajes de desviación estándar de longitud corporal durante el primer año de vida. Se grafican los resultados divididos en mujeres y varones comparados con las curvas SAP y OMS

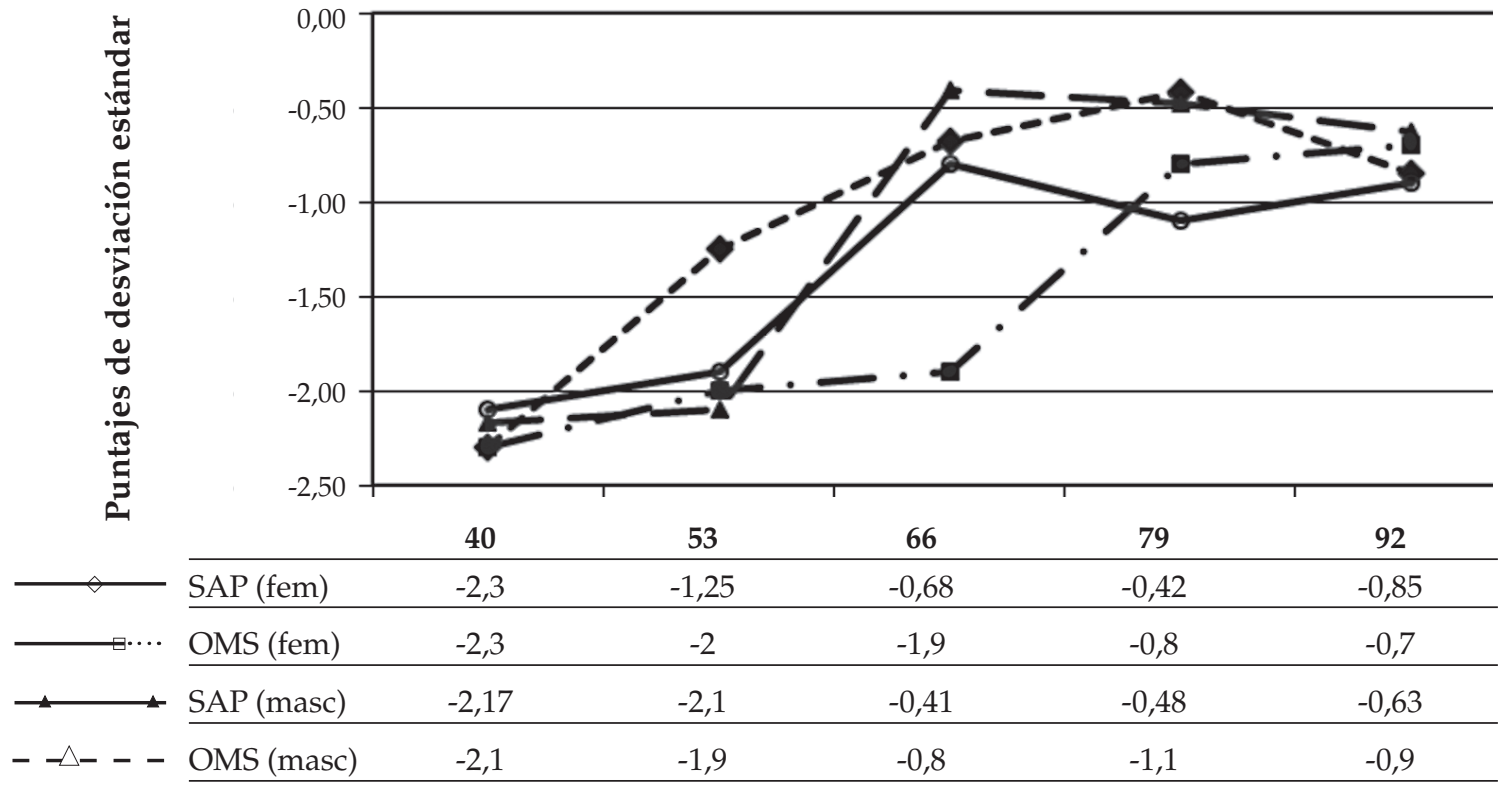

Edad posmenstrual 
TABla 3. Proporciones $(n, \%)$ de pacientes con peso y longitud corporal por debajo de 2 desviaciones estándar evaluados por el estándar SAP y OMS

\begin{tabular}{lcccccc}
\hline \multirow{2}{*}{$\begin{array}{l}\text { Edad corregida } \\
(n)\end{array}$} & Peso & \multicolumn{5}{c}{ Longitud corporal } \\
\cline { 2 - 7 } & SAP $n(\%)$ & OMS $n(\%)$ & OR (IC 95\%) & SAP $n(\%)$ & OMS $n(\%)$ & OR (IC 95\%) \\
\hline 40 sem. (204) & $34(17)$ & $35(17)$ & $0,97(0,57-1,62)$ & $104(51)$ & $107(52)$ & $0,93(0,62-1,40)$ \\
53 sem. (188) & $35(18)$ & $40(21)$ & $0,84(0,51-1,40)$ & $9(4,8)$ & $23(12,3)$ & $0,36(0,16-0,80)^{*}$ \\
66 sem. (144) & $15(10)$ & $20(14)$ & $0,72(0,35-1,47)$ & $52(36)$ & $44(31)$ & $1,28(0,78-2,11)$ \\
79 sem. (102) & $11(11)$ & $9(9)$ & $1,93(0,69-5,44)$ & $19(19)$ & $20(19)$ & $0,94(0,47-1,87)$ \\
92 sem. (190) & $29(15)$ & $27(14)$ & $1,09(0,62-1,92)$ & $34(18)$ & $29(16)$ & $1,21(0,70-2,09)$ \\
\hline
\end{tabular}

Puede implicar desde enfermedad cardiovascular, obesidad y diabetes tipo 1. Estos efectos fueron señalados inicialmente por Barker y corroborados por múltiples estudios observacionales y experimentales. ${ }^{8-10}$ La morbilidad posnatal relacionada con la prematuridad fue identificada también como asociación fuerte con retardo del crecimiento posnatal, aunque relacionada con enfermedades como la displasia broncopulmonar, la sepsis y la enterocolitis necrosante. La condición de la prematuridad también se ha asociado con una deuda nutricional durante el período neonatal inmediato, con repercusiones en la tasa de recanalización del crecimiento. ${ }^{11}$

Las curvas construidas por la OMS han tenido amplia difusión en nuestro medio y fueron adoptadas por la SAP. Este cambio dio lugar a varias publicaciones que analizaron las diferencias con las curvas nacionales, ${ }^{12-14}$ donde se observaba, en especial en los primeros meses de vida, un crecimiento mayor en peso en la muestra internacional en comparación con el estándar vigente. Pese a ello, se entendió que el carácter prescriptivo del estudio, caracterizado por niños sanos, con seguimiento pediátrico y vacunaciones completas, alimentados con pecho exclusivo, constituía una referencia de expresión del fenotipo en crecimiento en condiciones de salud adecuadas. ${ }^{15,16}$

En este contexto, creímos conveniente evaluar cuál era el efecto de adoptar estas curvas para la evaluación de una población de riesgo, como los RNMBPN, dado que una mayor proporción de niños con crecimiento posnatal subóptimo identificados por un cambio de curvas podría implicar intervenciones consideradas innecesarias con las curvas vigentes e incrementar el riesgo de sobrepeso más allá del año de vida.

Nuestro análisis muestra que solo al tercer mes de edad corregida la proporción de niños con peso subóptimo es mayor al ser analizadas por las curvas OMS, hallazgo similar al de los estudios previos a la adopción de las curvas. Este efecto podría atribuirse no solo a la prematuridad, sino además a la baja tasa de alimentación con pecho exclusivo en esta población y a la no existencia de fórmulas de continuación para esta población en nuestro medio. No observamos diferencias en la longitud corporal ni en la circunferencia craneal; consideramos que esto se debe, en gran medida, a la menor discrepancia entre las curvas, que en el caso del perímetro cefálico son casi idénticas.

La intervención nutricional durante el primer año de vida ha mostrado ser eficaz para mejorar no solo el crecimiento en peso ${ }^{17}$ y la longitud corporal más allá de la intervención, como lo muestran los estudios de Lucas, ${ }^{18}$ quien continuó siguiendo esa cohorte de prematuros alimentados hasta el noveno mes con fórmulas fortificadas, y comprobó la modificación del volumen del núcleo caudado y el desempeño en el grupo tratado con fórmulas con mayor densidad calórica ${ }^{19}$ al llegar a la adolescencia.

Los estudios recientes sugieren que continuar con fórmulas para prematuros (con mayor densidad calórico-proteica) podría ser beneficioso para estos niños. Sin embargo, el hecho debe ser analizado con cautela, dado que intervenciones nutricionales en este período podrían tener implicaciones en la prevalencia de la obesidad después del primer año. ${ }^{20,21}$ Asimismo, debemos reconocer que el estándar SAP que usamos desde la década de los setenta no contemplaba la obesidad como un problema prevalente en nuestra población. ${ }^{22}$

La OMS está abocada a la construcción de un patrón de crecimiento en prematuros con lineamientos prescriptivos, sobre la base de la disparidad de las evaluaciones de crecimiento en este grupo de niños de riesgo; ${ }^{23,24}$ sin embargo, hasta el presente los datos no se han publicado. Si bien hay informes que muestran algunos resultados sobre un emprendimiento de esta naturaleza, ${ }^{25,26}$ para nosotros persisten ciertas 
dudas sobre la factibilidad de obtener una población de prematuros "sanos" que puedan ser referencia, especialmente entre aquellos con edades menores de 28 semanas.

Nuestro estudio nos permite sugerir que el cambio de estándar para valorar el crecimiento durante el primer año de vida no implicaría una modificación significativa a nuestras prácticas de seguimiento desde las 40 semanas hasta el primer año de edad corregida.

Este estudio presenta como fortaleza una muestra con pacientes seguidos prospectivamente en un programa de seguimiento, con un observador entrenado en mediciones antropométricas, lo que disminuyó la variabilidad de las mediciones. Asimismo, el cumplimiento de las citaciones permitió no recurrir a la interpolación para evaluar el crecimiento en los puntos designados. Una debilidad es que representa una sola institución y sería conveniente un estudio multicéntrico para extender nuestras conclusiones.

Consideramos que el uso de estas curvas podría ser mejorado como herramienta de seguimiento, incorporando una escala de 40 a 92 semanas, asociada a los meses para la evaluación de la edad corregida, para el segmento de prematuros inscriptos en los planes de seguimiento.

\section{Agradecimientos}

A la Dra. Verónica Cravedi, que participó de las discusiones iniciales de este estudio y a quien recordamos con gran cariño.

\section{BIBLIOGRAFÍA}

1. Lejarraga H, Fustiñana C. Estándares de peso, longitud corporal y perímetro cefálico desde las 26 semanas hasta las 92 semanas de edad posmenstrual. Arch Argent Pediatr 1986;84:210-14.

2. Comité de Crecimiento y Desarrollo. Sociedad Argentina de Pediatría. Criterios de diagnóstico y tratamiento. Crecimiento y desarrollo. Buenos Aires:Sociedad Argentina de Pediatría; 1986.

3. Comité de Crecimiento y Desarrollo. Sociedad Argentina de Pediatría. Guías para la evaluación del crecimiento. $2^{a}$ Ed. Buenos Aires: Sociedad Argentina de Pediatría; 2001.

4. Fustiñana C, Ceriani Cernadas J, Lejarraga H. Influencia del retardo del crecimiento intrauterino y de la morbilidad neonatal sobre el crecimiento y la morbilidad postalta en niños con muy bajo peso al nacer. Arch Argent Pediatr 1987;85:223-32.

5. Fustiñana CA, Izbizky G, Rodríguez D, Mariani G, Ceriani Cernadas JM. Evaluación de la efectividad de un programa de Cuidado Intensivo Neonatal en niños de muy bajo peso al nacer. Impacto de la administración de surfactante. Arch Argent Pediatr 2009;107(1):9-15.

6. WHO Multicenter Growth Reference Study Group. Assessment of differences in linear growth among populations in the WHO Multicenter Growth Reference Study. Acta Paediatr 2006; (Suppl 450):56-65.
7. Sguassero Y,Moyano C, Aronna A, et al. Validación clínica de los nuevos estándares de crecimiento de la OMS: análisis de los resultados antropométricos en niños de 0 a 5 años de la ciudad de Rosario, Argentina. Arch Argent Pediatr 2008;106(3):198-204.

8. Baker DPJ. Mothers, babies and health in later life. Edimburgo: Livingstone Ed.; 1998.

9. Gluckman PD, Cutfield W, Hofman P, Hanson MA. The fetal, neonatal, and environments the long-term consecuences for disease risk. Early Human Development 2005;81:51-9.

10. Bloomfield FH, Oliver MH, Hawkins P, Holloway AC, et al. Periconceptional undernutrition in sheep accelerates maturation of the fetal hypothalamic-pituitary-adrenal axis in late gestation. Endocrinology 2004;145:4278-85.

11. Embleton NE, Pang N, Cooke RJ. Postnatal malnutrition and growth retardation: an inevitable consequence of current recommendations in pre-term infants? Pediatrics 2001;107:270-3.

12. Lejarraga $\mathrm{H}$. Referencias y estándares de crecimiento en la Argentina. Consideraciones del Grupo ad hoc para el análisis de las tablas de la Organización Mundial de la Salud y su uso en la Argentina. Arch Argent Pediatr 2007;105(2):159-66.

13. Lejarraga H. Consideraciones sobre el uso de tablas de crecimiento en la Argentina: Arch Argent Pediatr 2007;105:545-51.

14. Comité de Crecimiento y Desarrollo. La Sociedad Argentina de Pediatría actualiza las curvas de crecimiento de niñas y niños menores de 5 años. Arch Argent Pediatr 2008;106(5):462-7.

15. Abeyá Gilardon E. La salud y las curvas de crecimiento. Arch Argent Pediatr 2007;106(1):92-3.

16. Abeyá Gilardon E. Curvas de crecimiento nacionales, ¿dónde estamos y hacia dónde vamos? Arch Argent Pediatr 2003;101(5):350.

17. Tyson JE, Lasky RE, Mize CE, Richards CJ, et al. Growth, metabolic response, and development in very-low-birthweight infants fed banked human milk or enriched formula. I. Neonatal findings. J Pediatr 1983;103:95-104.

18. Lucas A, Gore SM, Cole TJ, Bamford MF, et al. Multicentre trial on feeding low birthweight infants: effects of diet on early growth. Arch Dis Child 1984;59:722-30.

19. Isaacs EB, Gadian DG, Sabatini S, Chong WK, et al. The effect of early human diet on caudate volumes and IQ. Pediatr Res 2008;63(3):608-14.

20. Ong KK, Ahmed ML,EmmettPM, etal. Association between postnatal catch-up growth and obesity in childhood: prospective cohort study. BMJ 2000;320:967-71.

21. Ong KK. Catch-up growth in small for gestational age babies: good or bad? Curr Opin Endocrinol Diabetes Obes 2007;14:30-4.

22. Cusminsky M, Castro E, Azcona LCh, et al. Estudio longitudinal del crecimiento desarrollo del niñoen La Plata. Comisión de Investigaciones, Científicas de la Provincia de Buenos Aires, La Plata; 1966.Págs.3-13.

23. de Onis M, Wijnhoven TMA, Onyango AW. Worldwide practices in child growth monitoring. J Pediatr 2004;144:461-5.

24. Villar J, Knight HE, de Onis M, Bertino E, et al; for the International Fetal and Newborn Growth Consortium (INTERGROWTH-21st). Conceptual issues related to the construction of prescriptive standards for the evaluation of postnatal growth of preterm infants. Arch Dis Child 2010;95(12):1034-8.

25. Bertino E, GilliG, Occhi L, Giuliani F, et al. Postnatal growth of preterm infants: which reference charts? Minerva Pediatr 2010;62(3 Suppl 1):71-4.

26. Bertino E, Di Nicola P, Giuliani F, Coscia A, et al. Evaluation of postnatal growth of preterm infants. J Matern Fetal Neonatal Med 2011;24(Suppl 2):9-11. 